\title{
A comparative review of fifty analytical representation of potential energy interaction for diatomic systems: One Hundred Years of History
}

\author{
Judith Araújo ${ }^{1}$ and Maikel Ballester ${ }^{1}$ \\ ${ }^{1}$ Universidade Federal de Juiz de Fora
}

September 16, 2020

\begin{abstract}
Interatomic potentials laid at the heart of molecular physics. They are a bridge between the spectroscopic and structural properties of molecular systems. In this paper, a century-old review from 1920 to 2020, of functional forms used to analytically represent potential energy as a function of interatomic distance for diatomic systems is presented. With such a purpose fifty functions were selected. For all of them, motivation and the main mathematical features are discussed. Our goal is to provide a chronological pathway to the reader, even with little knowledge on the subject, to understand how to calculate each parameter that composes the interatomic potentials, as well as obtain spectroscopic constants from them. Comparative evaluation for the $\mathrm{N}_{2}, \mathrm{CO}$, and $\mathrm{HeH}^{+}$systems in their ground electronic states are also presented.
\end{abstract}

\section{Hosted file}

IJQC_review.pdf available at https://authorea.com/users/359138/articles/481348-a-comparativereview-of-fifty-analytical-representation-of-potential-energy-interaction-for-diatomicsystems-one-hundred-years-of-history 